\title{
ENSAIOS
}

\section{ACONSELHAMENTO PARA ATIVIDADE FÍSICA NA ATENÇÃO PRIMÁRIA À SAÚDE: UMA REVISÃO INTEGRATIVA}

\author{
COUNSELING FOR PHYSICAL ACTIVITY IN PRIMARY HEALTH CARE: \\ AN INTEGRATIVE REVIEW C
}

\section{ASESORÍA PARA LA ACTIVIDAD FÍSICA EN LA ATENCIÓN PRIMARIA DE SALUD: UNA REVISIÓN INTEGRATIVA Cे}

doi" https://doi.org/10.22456/1982-8918.104360

João Miguel de Souza Neto*<miguel.edf@hotmail.com>

(iD) Geraldo Eduardo Guedes de Brito*<eduardo.guedes.ufpb@gmail.com>

Mathias Roberto Loch** <mathiasuel@hotmail.com>

Sanderson Soares da Silva* <sandersonjf@gmail.com>

Filipe Ferreira da Costa*<filipefcosta@outlook.com>

\footnotetext{
*Universidade Federal da Paraíba. Centro de Ciência da Saúde, Departamento de Educação Física. João Pessoa, PB, Brasil.

**Universidade Estadual de Londrina. Londrina, PR, Brasil.
}

\begin{abstract}
Resumo: O objetivo foi sintetizar a produção acadêmica sobre a temática do aconselhamento para atividade física na atenção primária à saúde do Sistema Único de Saúde (SUS). Trata-se de revisão integrativa da literatura, realizada nas bases LILACS, MEDLINE, PubMed e SciELO, publicada até dezembro de 2018. Foram analisados 22 estudos, dos quais 13 foram observacionais, sete intervenções e dois qualitativos. A prevalência de aconselhamento para usuários variou de $20 \%$ a $59,4 \%$ entre os estudos e observou-se o perfil de usuários que mais recebem aconselhamento (mulheres, pessoas mais velhas e com diagnóstico de doenças crônicas). Os profissionais mais aconselhadores são: médicos, aqueles fisicamente ativos, que se sentem capazes de aconselhar e que não identificam a falta de tempo como barreira. As intervenções apresentaram efeitos modestos nos níveis de atividade física dos usuários e nas práticas de aconselhamento dos profissionais.
\end{abstract}

Palavras chave: Aconselhamento. Educação em saúde. Atenção primária à saúde. Atividade motora.
Recebido em: 10-06-2020

Aprovado em: 30-08-2020 Publicado em: 27-11-2020

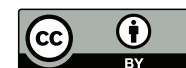

Este é um artigo publicado sob a licença Creative Commons Atribuição 4.0 Internacional (CC BY 4.0).

eISSN: 1982-8918 


\section{INTRODUÇÃO}

A Estratégia de Saúde da Família (ESF), modelo prioritário de organização da atenção primária no Sistema Único de Saúde (SUS), ampliou significativamente o acesso aos serviços de saúde. Com sua criação, encontrou-se espaço institucional para o desenvolvimento de ações de educação e promoção da saúde, importantes para a mudança de paradigma assistencial (BRASIL, 2017). Essas ações, no âmbito da atenção primária, são estratégicas para a promoção de modos de vida saudáveis, particularmente para agir sobre os principais fatores de risco modificáveis, como o tabagismo, etilismo, dieta inadequada e inatividade física, que contribuem para o perfil de morbimortalidade brasileiro (SCHMIDT et al., 2011). Dentre as ações de educação e promoção da saúde alinhadas com o processo de trabalho na ESF, destaca-se o aconselhamento.

No contexto do cuidado em saúde, o aconselhamento é compreendido como um processo de escuta ativa, individualizado e centrado no usuário, que pressupõe a capacidade de estabelecer uma relação de confiança entre os interlocutores, visando ao resgate dos recursos internos do usuário para que ele mesmo tenha possibilidade de reconhecer-se como sujeito de sua própria saúde e transformação (BRASIL, 1999). Particularmente para o comportamento atividade física, pode ser compreendido como uma orientação geral e estruturada voltada ao incentivo para a prática de atividade física em diferentes domínios (FLORINDO; ANDRADE, 2015), sendo apresentado como uma estratégia de custo relativo baixo, que pode ser realizada por qualquer profissional da saúde, demandando poucos minutos de um atendimento rotineiro (NICE, 2013).

No Brasil, há um crescente interesse dos pesquisadores no sentido de conhecer diferentes aspectos do aconselhamento para atividade física no SUS, como sua prevalência (SIQUEIRA, 2016), seus fatores associados (BARBOSA et al., 2017), o nível de conhecimento dos profissionais (BURDICK et al., 2015), bem como o efeito de intervenções nas práticas de aconselhamento dos profissionais (MENDONÇA; TOLEDO; LOPES, 2015) e no nível de atividade física dos usuários de saúde (RIBEIRO et al., 2017). Tendo em vista que o Brasil possui um dos maiores sistemas universais de saúde do mundo e uma organização singular da atenção primária à saúde (ALY et al., 2017), a variedade de definições operacionais e de correlatos do aconselhamento utilizados nesses estudos, bem como a diversidade de objetivos e abordagens das intervenções realizadas, pode dificultar a compreensão global desta estratégia de cuidado em saúde, distanciando o conhecimento produzido dos contextos concretos de produção de saúde.

Por outro lado, a síntese e o debate sobre o conhecimento produzido em relação ao aconselhamento para atividade física podem contribuir para a qualificação das práticas e o maior envolvimento dos profissionais na realização de ações de promoção e educação em saúde. Portanto, este estudo teve como objetivo sintetizar a produção acadêmica sobre a temática do aconselhamento para atividade física na atenção primária à saúde do SUS.

\section{MÉTODO}

Este estudo compreende uma revisão integrativa sobre o aconselhamento para atividade física na atenção primária à saúde do SUS. Optou-se por esta 
modalidade de pesquisa por se tratar de uma ferramenta que proporciona uma síntese do estado da arte, além de permitir a inclusão de estudos com objetivos e desenhos metodológicos heterogêneos, auxiliando uma compreensão global sobre o tema e suas implicações para a prática profissional (SOUZA; SILVA; CARVALHO, 2010). Estabeleceram-se as seguintes perguntas norteadoras: "Quais as produções científicas disponíveis sobre práticas de aconselhamento para a atividade física na atenção primária à saúde do SUS? Quais as fragilidades e potencialidades para a adoção desta prática no processo de trabalho na atenção primária à saúde do SUS?".

Para respondê-las, realizou-se uma busca na literatura científica, sendo considerados os seguintes critérios de elegibilidade: texto completo, escritos nos idiomas inglês, espanhol ou português; estudos desenvolvidos no Brasil; publicações com a temática do aconselhamento/orientação de atividade física na atenção primária.

A pesquisa foi realizada nas bases de dados da Literatura Latino-Americana e do Caribe em Ciências da Saúde (LILACS); Medical Literature Analysis and Retrieval System Online (MEDLINE); Publisher Medline (PubMed); na biblioteca digital Scientific Electronic Library On-line (SciELO) e no Google Acadêmico. Buscando-se evitar a perda de informações relevantes, foi feita busca complementar nas listas de referências dos trabalhos incluídos e em periódicos não indexados com reconhecida produção na área.

Os critérios de exclusão foram: artigos de revisão da literatura, dissertações, teses ou capítulos de livros, artigos que não abordassem o aconselhamento para atividade física como variável dependente ou exposição principal. A pesquisa nas bases de dados foi feita de outubro a dezembro de 2018, sendo incluídas todas as publicações até o final deste período. Utilizaram-se os descritores, controlados e combinados com operadores booleanos: promoção da saúde (health promotion) OR aconselhamento (counselling) OR educação para saúde (health education); $A N D$ atividade física (physical activity) OR exercício (exercise); AND Sistema Único de Saúde - SUS (unfied health system) OR atenção primária à saúde (primary health care) OR estratégia saúde da família (family health strategy); AND Brasil (Brazil).

A seleção dos artigos foi realizada independentemente, por dois autores, e um consenso com um terceiro autor foi realizado nos casos de diferenças. Visando à sistematização dos dados, os autores extraíram dados referentes ao estudo e características metodológicas.

\section{RESULTADOS}

Excluídas duplicatas entre as bases de dados $(n=41)$, o procedimento de buscas eletrônicas recuperou 2.010 artigos potencialmente relevantes. Após a leitura dos títulos e resumos, 52 artigos foram selecionados para leitura na íntegra, dos quais 19 foram incluídos. Os artigos foram excluídos por: incongruência com a temática de práticas educativas e de aconselhamento para atividade física $(n=18)$; não realizados na atenção primária à saúde $(n=6)$; ensaios teóricos $(n=3)$; pesquisa não conduzida no país $(n=6)$. Posteriormente, oito estudos foram localizados nas referências dos artigos e adicionados à revisão por atenderam aos critérios de elegibilidade, totalizando 22 estudos.

No Quadro 1 são apresentadas as informações gerais dos estudos observacionais incluídos na revisão $(n=13)$. O público-alvo das investigações, em sua 
maioria, foi constituído de usuários das unidades básicas de saúde (BARBOSA et al., 2017; HÄFELE; SIQUEIRA, 2016; LOPES et al., 2014; SANTOS et al., 2012; TOLEDO; ABREU; LOPES, 2013; TOLEDO et al., 2017), com três estudos de base domiciliar (DURO et al., 2015; HALLAL et al., 2010; SIQUEIRA et al., 2009). A abrangência dos estudos variou entre amostras selecionadas em apenas uma unidade de saúde (SANTOS et al., 2012; TOLEDO; ABREU; LOPES, 2013) até amostragem de domicílios em 100 cidades brasileiras (DURO et al., 2015), bem como amostragem de linhas telefônicas de unidades básicas de saúde de todo o Brasil (FLORINDO et al., 2013).

A operacionalização da variável aconselhamento para atividade física variou entre os estudos, notadamente quanto ao responsável pelo aconselhamento e a referência temporal. A maioria dos estudos se referiu aos profissionais de saúde que compõem as unidades de saúde (BARBOSA et al., 2017; DURO et al., 2015; HÄFELE; SIQUEIRA, 2016; LOPES et al., 2014; SANTOS et al., 2012; TOLEDO; ABREU; LOPES, 2013), enquanto no estudo de HALLAL et al. (2010) não foi especificado quem seria o aconselhador. Alguns estudos investigaram o recebimento de aconselhamento por toda a vida (DURO et al., 2015; FLORINDO et al., 2015; LOPES et al., 2014; SIQUEIRA et al., 2009; TOLEDO; ABREU; LOPES, 2013), outros não especificaram (BARBOSA et al., 2017; HALLAL et al., 2010; SANTOS et al., 2012), e um determinou um período específico (últimos 12 meses) (DURO et al., 2015). Além da atividade física, quatro estudos incluíram a alimentação na pergunta sobre a realização do aconselhamento (LOPES et al., 2014; SANTOS et al., 2012; TOLEDO; ABREU; LOPES, 2013; TOLEDO et al., 2017).

Quanto à mensuração do aconselhamento realizado pelos profissionais, optou-se pelo uso dos estágios de mudança de comportamento, sendo considerados profissionais de saúde "aconselhadores" aqueles que realizavam tal prática há mais de seis meses (FLORINDO et al., 2015; FLORINDO et al., 2014; FLORINDO et al., 2013). As prevalências de aconselhamento relatado pelos usuários variaram de $20 \%$ em amostra de adultos de 100 cidades brasileiras (DURO et al., 2015) a $59,4 \%$ em usuários diabéticos atendidos em unidades básicas de saúde do estado de Pernambuco (BARBOSA et al., 2017). Os estudos analisaram os correlatos do recebimento de aconselhamento, e, de maneira geral, encontraram associação positiva com o sexo feminino (BARBOSA et al., 2017; DURO et al., 2015), mais velhos (DURO et al., 2015; HÄFELE; SIQUEIRA, 2016; HALLAL et al., 2010), maior status socioeconômico (DURO et al., 2015; HALLAL et al., 2010), presença de excesso de peso e fatores de risco cardiovasculares (BARBOSA et al., 2017; DURO et al., 2015; HALLAL et al., 2010), presença de doenças crônicas (DURO et al., 2015; HÄFELE; SIQUEIRA, 2016; LOPES et al., 2014; SANTOS et al., 2012; SIQUEIRA et al., 2009), uso contínuo de medicamento (HÄFELE; SIQUEIRA, 2016; SIQUEIRA et al., 2009), tabagismo (DURO et al., 2015; SIQUEIRA et al., 2009) e inatividade física (HALLAL et al., 2010; SIQUEIRA et al., 2009). Entre os médicos e enfermeiros, o aconselhamento esteve associado à realização de medidas da atividade física dos usuários, sentir-se preparado para aconselhar, a percepção de disponibilidade de tempo e a existência de programas de atividade física nas unidades de saúde (FLORINDO et al., 2013), enquanto entre os agentes comunitários de saúde o aconselhamento foi associado ao seu nível de atividade física (FLORINDO et al., 2015). No Quadro 1 são apresentadas as informações gerais dos estudos observacionais incluídos na revisão $(n=13)$. 
Quadro 1 - Estudos observacionais sobre aconselhamento para atividade física na atenção primária à saúde no Brasil segundo o autor, ano, amostra, operacionalização da variável e principais resultados.

\begin{tabular}{|c|c|c|c|}
\hline Autor, ano & Amostra & Operacionalização da variável aconselhamento & Principais resultados \\
\hline $\begin{array}{l}\text { Barbosa et } \\
\text { al., } 2017\end{array}$ & $\begin{array}{l}785 \text { hipertensos e } 822 \\
\text { diabéticos de } 208 \text { UBS de } \\
35 \text { municípios do Estado de } \\
\text { Pernambuco. }\end{array}$ & $\begin{array}{l}\text { Algum profissional da Estratégia Saúde da Família já } \\
\text { te aconselhou a modificar (melhorar) seus hábitos de } \\
\text { atividade física? }\end{array}$ & $\begin{array}{l}\text { - Prevalência nos usuários hipertensos: } 53,0 \% \text {; } \\
\text { - Prevalência nos usuários diabéticos: } 59,4 \% \text {; } \\
\text { - O recebimento de aconselhamento foi associado ao } \\
\text { excesso de peso e/ou fatores de risco cardiovascular. }\end{array}$ \\
\hline $\begin{array}{l}\text { Duro et al., } \\
2015\end{array}$ & $\begin{array}{l}12.402 \text { adultos de } 100 \text { cidades } \\
\text { por amostragem de domicílio. }\end{array}$ & $\begin{array}{l}\text { Alguma vez na vida, em uma consulta no Centro } \\
\text { de Saúde, algum profissional (médico, enfermeiro, } \\
\text { nutricionista...) Ihe disse que o(a) Sr.(a) deveria } \\
\text { melhorar/mudar sua alimentação e/ou fazer atividade } \\
\text { física para melhorar a sua saúde? }\end{array}$ & $\begin{array}{l}\text { - Prevalência nos usuários: } 20 \% \text {; } \\
\text { - O recebimento de aconselhamento foi associado às } \\
\text { mulheres, pessoas mais velhas, casadas, com maior } \\
\text { status socioeconômico, fumantes, fisicamente ativos } \\
\text { e relatando hipertensão, diabetes e excesso de peso } \\
\text { corporal. }\end{array}$ \\
\hline $\begin{array}{l}\text { Hafele e } \\
\text { Siqueira, } \\
2016\end{array}$ & $\begin{array}{l}525 \text { usuários de } 35 \text { UBS do } \\
\text { município de Pelotas, RS. }\end{array}$ & $\begin{array}{l}\text { Durante o último ano, em alguma vez em que o(a) Sr.(a) } \\
\text { esteve na UBS, recebeu aconselhamento para praticar } \\
\text { atividade física? }\end{array}$ & $\begin{array}{l}\text { - Prevalência nos usuários: } 35,4 \% \text {, sendo realizado } \\
\text { por médicos ( } 80 \%) \text {, de forma verbal }(97,2 \%) \text {, com o } \\
\text { conteúdo principal a indicação de caminhada }(70,3 \%) \text { e } \\
\text { atividades físicas no lazer }(84,9 \%) ; \\
\text { - Receber aconselhamento foi associado com } \\
\text { maior idade, com diagnóstico referido por médico } \\
\text { de hipertensão arterial, que faziam uso contínuo de } \\
\text { medicamentos e que visitaram mais vezes a unidade de } \\
\text { saúde no último ano. }\end{array}$ \\
\hline $\begin{array}{l}\text { Hallal et } \\
\text { al., } 2010\end{array}$ & $\begin{array}{l}972 \text { adultos selecionados por } \\
\text { amostragem de domicílio no } \\
\text { município de Pelotas, RS. }\end{array}$ & Alguém já te recomendou fazer atividade física? & $\begin{array}{l}\text { - Prevalência nos usuários: } 56,2 \% \text {; } \\
\text { - Receber aconselhamento foi associado a maior idade, } \\
\text { maior nível socioeconômico, excesso de peso corporal e } \\
\text { inatividade física. }\end{array}$ \\
\hline $\begin{array}{l}\text { Lopes et } \\
\text { al., } 2014\end{array}$ & $\begin{array}{l}1.616 \text { usuários de } 4 \text { UBS do } \\
\text { município de Belo Horizonte, } \\
\text { MG. }\end{array}$ & $\begin{array}{l}\text { Alguma vez na vida, em uma consulta no Centro } \\
\text { de Saúde, algum profissional (médico, enfermeiro, } \\
\text { nutricionista...) Ihe disse que o(a) Sr.(a) deveria } \\
\text { melhorar/mudar sua alimentação e/ou fazer atividade } \\
\text { física para melhorar a sua saúde? }\end{array}$ & $\begin{array}{l}\text { - Prevalência nos usuários: } 52 \% \text {; } \\
\text { - O recebimento de aconselhamento foi associado } \\
\text { à presença de agravos à saúde e a participação no } \\
\text { Programa Academia da Saúde. }\end{array}$ \\
\hline
\end{tabular}

Continua na próxima página..

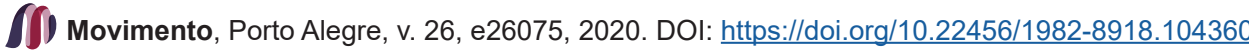


Continuação do quadro 1

\begin{tabular}{|c|c|c|c|}
\hline $\begin{array}{l}\text { Santos et } \\
\text { al., } 2012\end{array}$ & $\begin{array}{l}499 \text { usuários de uma UBS do } \\
\text { município de Belo Horizonte, } \\
\text { MG. }\end{array}$ & $\begin{array}{l}\text { Você já foi orientado durante algum atendimento } \\
\text { individual ou em atividades coletivas desenvolvidas na } \\
\text { UBS acerca da prática da alimentação saudável e/ou de } \\
\text { atividade física? }\end{array}$ & $\begin{array}{l}\text { - Prevalência dos usuários: } 59,3 \% \text {; } \\
\text { - O aconselhamento foi mais frequente entre os usuários } \\
\text { com maior número de morbidades e as principais foram } \\
\text { a falta de tempo e a necessidade de mudança de } \\
\text { hábitos. }\end{array}$ \\
\hline $\begin{array}{l}\text { Santos et } \\
\text { al., } 2015\end{array}$ & $\begin{array}{l}30 \text { ACS de uma UBS com } \\
\text { ESF no distrito de Ermelino } \\
\text { Matarazzo, zona leste de São } \\
\text { Paulo. }\end{array}$ & $\begin{array}{l}\text { Avaliado por uma pergunta contendo seis categorias } \\
\text { de resposta baseadas nos estágios de mudança de } \\
\text { comportamento, sendo considerados profissionais de } \\
\text { saúde "aconselhadores" aqueles que realizam tal prática } \\
\text { há mais de seis meses. }\end{array}$ & $\begin{array}{l}\text { - Metade das ACS recomendava atividade física para } \\
\text { os usuários e colaborava ou participava de grupos de } \\
\text { promoção da atividade física desenvolvidos nas UBS. }\end{array}$ \\
\hline $\begin{array}{l}\text { Siqueira et } \\
\text { al., } 2009\end{array}$ & $\begin{array}{l}1.940 \text { adultos e } 1.891 \text { idosos } \\
\text { na Região Sul e } 2.120 \text { adultos } \\
\text { e } 2.112 \text { idosos na Região } \\
\text { Nordeste do Brasil. }\end{array}$ & $\begin{array}{l}\text { Alguma vez na vida, em uma consulta no [Posto de } \\
\text { Saúde de Abrangência], algum médico lhe disse que } \\
\text { o(a) Sr.(a) deveria fazer exercícios para melhorar a sua } \\
\text { saúde? }\end{array}$ & $\begin{array}{l}\text { - Prevalência nos usuários adultos: } 28,9 \% \text {; } \\
\text { - Prevalência nos usuários idosos: } 38,9 \% \text {; } \\
\text { - O recebimento de aconselhamento foi associado às } \\
\text { mulheres, nível socioeconômico mais baixo, fumantes, } \\
\text { inativos, diagnóstico de doenças crônicas e uso de } \\
\text { medicação contínua. }\end{array}$ \\
\hline $\begin{array}{l}\text { Toledo; } \\
\text { Abreu; } \\
\text { Lopes, } \\
2013\end{array}$ & $\begin{array}{l}417 \text { usuários de uma UBS do } \\
\text { município de Belo Horizonte, } \\
\text { MG. }\end{array}$ & $\begin{array}{l}\text { Alguma vez na vida, em uma consulta no Centro } \\
\text { de Saúde, algum profissional (médico, enfermeiro, } \\
\text { nutricionista...) Ihe disse que o(a) Sr.(a) deveria } \\
\text { melhorar/mudar sua alimentação e/ou fazer atividade } \\
\text { física para melhorar a sua saúde? }\end{array}$ & $\begin{array}{l}\text { - Prevalência nos usuários: 40,8 \%; } \\
\text { - A adesão a modos de vida saudáveis foi associada } \\
\text { à participação no programa Academia da Cidade e } \\
\text { considerar a própria alimentação saudável. }\end{array}$ \\
\hline $\begin{array}{l}\text { Toledo et } \\
\text { al., } 2017\end{array}$ & $\begin{array}{l}417 \text { usuários de uma UBS do } \\
\text { município de Belo Horizonte, } \\
\text { MG. }\end{array}$ & $\begin{array}{l}\text { Alguma vez na vida, em uma consulta no Centro } \\
\text { de Saúde, algum profissional (médico, enfermeiro, } \\
\text { nutricionista...) Ihe disse que o(a) Sr.(a) deveria } \\
\text { melhorar/mudar sua alimentação e/ou fazer atividade } \\
\text { física para melhorar a sua saúde? }\end{array}$ & $\begin{array}{l}\text { - Prevalência nos usuários: 40,8 \%; } \\
\text { - Associados à maior frequência de recebimento de } \\
\text { aconselhamento estiveram: indivíduos com hipertensão } \\
\text { arterial e hipercolesterolêmica, com consumo adequado } \\
\text { de temperos ultra processados e participantes das } \\
\text { atividades do Programa Academia da Saúde. }\end{array}$ \\
\hline $\begin{array}{l}\text { Flores et } \\
\text { al., } 2018\end{array}$ & $\begin{array}{l}1.281 \text { idosos residentes na zona } \\
\text { urbana do município de Pelotas, } \\
\text { RS. }\end{array}$ & $\begin{array}{l}\text { Desde [mês] do ano passado até agora, algum } \\
\text { profissional de saúde orientou o(a) Sr.(a) a...", } \\
\text { complementado por "reduzir o consumo de sal?", "reduzir } \\
\text { o consumo de açúcar e doces?", "reduzir o consumo de } \\
\text { gorduras?" e "praticar atividade física? }\end{array}$ & $\begin{array}{l}\text { - Idosos que referiram receber orientaçães de } \\
\text { profissionais de saúde relataram redução do consumo } \\
\text { de sal e de açúcar e doces e maior prática de atividade } \\
\text { física, em comparação aos idosos que não receberam } \\
\text { orientação. }\end{array}$ \\
\hline
\end{tabular}

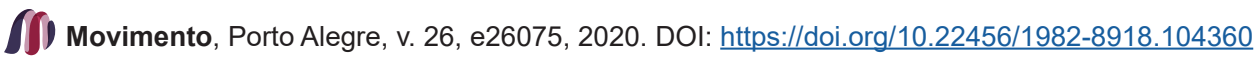


Continuação do quadro 1.

\begin{tabular}{|c|c|c|c|}
\hline $\begin{array}{l}\text { Florindo et } \\
\text { al., } 2013\end{array}$ & $\begin{array}{l}347 \text { enfermeiros e } 182 \\
\text { médicos de UBS selecionadas } \\
\text { aleatoriamente no território } \\
\text { nacional. }\end{array}$ & $\begin{array}{l}\text { Avaliado por uma pergunta contendo seis categorias } \\
\text { de resposta baseadas nos estágios de mudança de } \\
\text { comportamento, sendo considerados profissionais de } \\
\text { saúde "aconselhadores" aqueles que realizam tal prática } \\
\text { há mais de seis meses. }\end{array}$ & $\begin{array}{l}\text { - Prevalência total nos profissionais de saúde: } 68,9 \% \text {; } \\
\text { - Tiveram mais chances de aconselhar os profissionais } \\
\text { que: avaliam a atividade física dos usuários; não } \\
\text { referem a falta de tempo como uma barreira para o } \\
\text { aconselhamento; sentem-se preparados para fornecer } \\
\text { aconselhamento; e trabalham em UBS que oferecem } \\
\text { programas de atividade física para usuários. }\end{array}$ \\
\hline
\end{tabular}

Fonte: Os autores 
No Quadro 2 são apresentados os estudos de intervenção incluídos na revisão ( $n=7)$. Os estudos analisados objetivaram avaliar o efeito de intervenções educativas nas práticas, percepções e nível de conhecimento sobre aconselhamento para atividade física de profissionais de saúde (FIGUEIRA et al., 2015; FLORINDO et al., 2014; MENDONÇA; TOLEDO; LOPES, 2015; SÁ; FLORINDO, 2012), bem como o efeito de ações de aconselhamento nos níveis de atividade física e nos estágios de mudança de comportamento dos usuários (GOMES; DUARTE, 2008; RIBEIRO et al., 2017; SÁ; FLORINDO, 2012). A maioria dos estudos incluiu um grupo controle na intervenção (COSTA et al., 2015; FIGUEIRA et al., 2015; FLORINDO et al., 2014; GOMES; DUARTE, 2008; RIBEIRO et al., 2017) e um deles testou mais de um tipo de intervenção (educação vs aulas com exercícios) (RIBEIRO et al., 2017). A duração das intervenções variou de três (GOMES; DUARTE, 2008) a 12 meses (RIBEIRO et al., 2017), com acompanhamento pós-intervenção de seis meses em dois estudos (COSTA et al., 2015; RIBEIRO et al., 2017).

Nas intervenções direcionadas aos usuários, a duração variou de três encontros mensais de uma hora, interpassados por visitas domiciliares de dez minutos (GOMES; DUARTE, 2008) até 32 horas organizadas em 16 encontros de duas horas durante 12 meses (RIBEIRO et al., 2017). As principais estratégias de intervenção para os usuários foram visitas domiciliares (COSTA et al., 2015), encontros na UBS (RIBEIRO et al., 2017) ou combinação destas (GOMES; DUARTE, 2008). Os responsáveis pelo desenvolvimento das ações foram os agentes comunitários de saúde (COSTA et al., 2015; GOMES; DUARTE, 2008) e profissionais de saúde externos ao serviço (GOMES; DUARTE, 2008; RIBEIRO et al., 2017). Os desfechos investigados foram os níveis de atividade física (COSTA et al., 2015; GOMES; DUARTE, 2008; RIBEIRO et al., 2017) e estágios de mudança de comportamento para a prática de atividade física (COSTA et al., 2015; GOMES; DUARTE, 2008).

As estratégias incluíram rodas de conversa, oficinas, jogos educativos, práticas corporais alternativas e de autocuidado (COSTA et al., 2015; FIGUEIRA et al., 2015; FLORINDO et al., 2014; MENDONÇA; TOLEDO; LOPES, 2015; SÁ; FLORINDO, 2012). O arcabouço da teoria pedagógica de Paulo Freire foi utilizado na maioria das intervenções (GOMES; DUARTE, 2008; FIGUEIRA et al., 2015; FLORINDO et al., 2014; SÁ; FLORINDO, 2012). Os dois estudos qualitativos incluídos na revisão envolveram análises do discurso de profissionais de saúde participantes de intervenções educativas para a promoção do aconselhamento para atividade física. No estudo de Sá, Velardi e Florindo (2016) foram identificados cinco limites e potencialidades para a educação sobre atividade física na Estratégia de Saúde da Família. Os cinco temas foram identificados e discutidos: organização do trabalho e educação no trabalho; relação dos profissionais com a atividade física; ponto de vista dos profissionais sobre o processo saúde-doença e sobre aconselhamento; falta de cuidados para os profissionais de saúde; e avaliação dos elementos-chave da estratégia pedagógica. Já no estudo de Florindo et al. (2018) foram destacados positivamente a abordagem do curso focado na melhora da qualidade de vida e bemestar dos pacientes, prevenção de doenças e melhorias na saúde. Como aspectos que podem melhorar, apontaram-se a necessidade de experiências práticas com atividade física entre os próprios profissionais e a necessidade de articular as ações de aconselhamento com as oportunidades locais de programas de atividade física. No Quadro 2 são apresentados os estudos de intervenção incluídos na revisão $(n=7)$. 
Quadro 2 - Estudos de intervenção sobre aconselhamento para atividade física na atenção primária à saúde no Brasil segundo o autor, ano, caracterização da amostra, objetivo da intervenção e principais resultados.

\begin{tabular}{|c|c|c|c|}
\hline Autor/ano & Caracterização da amostra & Objetivo da intervenção & Principais resultados \\
\hline $\begin{array}{l}\text { Gomes et } \\
\text { al., } 2008^{20}\end{array}$ & $\begin{array}{l}\text { GI }=51 \text { e } \mathrm{GC}=52 \\
\text { de duas unidades básicas } \\
\text { de saúde de Florianópolis, } \\
\text { SC }\end{array}$ & $\begin{array}{l}\text { Implementar e avaliar uma intervenção de } \\
\text { aconselhamento para atividade física por meio } \\
\text { de visitas domiciliares e palestras para usuários } \\
\text { atendidos em uma unidade básica de saúde. }\end{array}$ & $\begin{array}{l}\text { O GI melhorou a percepção de saúde e aumentou o nível de } \\
\text { atividade física total. }\end{array}$ \\
\hline $\begin{array}{l}\text { Costa et } \\
\text { al., } 2015^{28}\end{array}$ & $\begin{array}{l}\mathrm{GI}=90 \text { e } \mathrm{GC}=86 \text { de duas } \\
\text { unidades básicas de saúde } \\
\text { de São Paulo, SP }\end{array}$ & $\begin{array}{l}\text { Verificar a efetividade de uma intervenção } \\
\text { educativa com ACS para promover atividade física } \\
\text { por meio de visitas domiciliares aos usuários } \\
\text { atendidos em uma unidade básica de saúde. }\end{array}$ & $\begin{array}{l}\text { Não foram observadas mudanças no nível de atividade física } \\
\text { e nos estágios de mudança de comportamento em usuários. }\end{array}$ \\
\hline $\begin{array}{l}\text { Ribeiro et } \\
\text { al., } 2015^{21}\end{array}$ & $\begin{array}{l}\text { - Gl exercício físico n=54 } \\
\text { - Gl educação em saúde } \\
\text { n=54 } \\
\text { - GC n= } 49 \\
\text { - Local: São Paulo, SP }\end{array}$ & $\begin{array}{l}\text { Avaliar o efeito de dois tipos de intervenções nos } \\
\text { níveis de atividade física de usuários atendidos em } \\
\text { duas unidades básicas de saúde. }\end{array}$ & $\begin{array}{l}\text { Após } 12 \text { meses, ambos os grupos de intervenção } \\
\text { aumentaram a prática de atividade física. No entanto, } \\
\text { observou-se que a intervenção de educação em saúde foi } \\
\text { mais efetiva para a manutenção da prática de atividade física } \\
\text { no período pós-intervenção. }\end{array}$ \\
\hline $\begin{array}{l}\text { Sá et al., } \\
2012^{26}\end{array}$ & $\begin{array}{l}-G I n=39 \\
-G C n=44\end{array}$ & $\begin{array}{l}\text { Avaliar os efeitos de um programa educativo sobre } \\
\text { práticas e saberes de trabalhadores da ESF para a } \\
\text { promoção de atividade física. }\end{array}$ & $\begin{array}{l}\text { A partir da análise qualitativa, foi possível identificar } \\
\text { alterações nas representações do GI sobre a atividade física } \\
\text { e sobre a qualidade do aconselhamento para a prática. }\end{array}$ \\
\hline $\begin{array}{l}\text { Florindo et } \\
\text { al., } 2014^{19}\end{array}$ & $\begin{array}{l}\text { - GI n=65 } \\
\text { - GC n=30 } \\
\text { - Local: São Paulo, SP }\end{array}$ & $\begin{array}{l}\text { Descrever uma metodologia de treinamento para } \\
\text { fornecer aconselhamento sobre atividade física em } \\
\text { ACS que atuam na atenção primária à saúde. }\end{array}$ & $\begin{array}{l}\text { Observou-se um aumento significativo no conhecimento } \\
\text { sobre recomendações de atividade física dos ACS no GI } \\
\text { comparado ao GC. }\end{array}$ \\
\hline $\begin{array}{l}\text { Figueira et } \\
\text { al., } 2015^{27}\end{array}$ & $\begin{array}{l}\text { - } 22 \text { profissionais de saúde } \\
\text { - Local: Belo Horizonte, MG }\end{array}$ & $\begin{array}{l}\text { Comparar a percepção de profissionais de um } \\
\text { centro de saúde sobre o aconselhamento de } \\
\text { modos de vida saudáveis antes e depois de uma } \\
\text { intervenção educativa. }\end{array}$ & $\begin{array}{l}\text { Observaram-se poucas mudanças nas percepções dos } \\
\text { profissionais após a intervenção educativa. Identificou- } \\
\text { se que, após a intervenção educativa, houve maior } \\
\text { sensibilização para a educação em saúde por meio de } \\
\text { práticas multiprofissionais. }\end{array}$ \\
\hline $\begin{array}{l}\text { Mendonça; } \\
\text { Toledo; } \\
\text { Costa, } \\
2015^{18}\end{array}$ & $\begin{array}{l}\text { - } 66 \text { profissionais de saúde } \\
\text { - Local: Belo Horizonte, MG }\end{array}$ & $\begin{array}{l}\text { Analisar o efeito de ações educativas realizadas } \\
\text { com profissionais da Atenção Primária sobre o } \\
\text { aconselhamento sobre modos saudáveis de vida. }\end{array}$ & $\begin{array}{l}\text { Verificou-se aumento da prática de aconselhamento sobre } \\
\text { modos de vida saudáveis }(p=0,009) \text {. Os profissionais } \\
\text { relataram acreditar que a prática regular de atividade física } \\
(95,5 \%) \text { e a alimentação saudável }(98,5 \%) \text { são capazes de } \\
\text { influenciar a saúde, sobretudo a qualidade de vida. }\end{array}$ \\
\hline
\end{tabular}

Fonte: Os autores

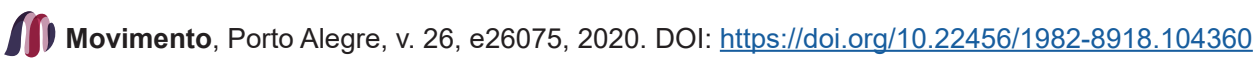




\section{DISCUSSÃO}

O presente estudo objetivou sumarizar e analisar os estudos que reportaram as práticas do aconselhamento para atividade física na atenção primária à saúde no Brasil. A maioria dos estudos analisou o recebimento/oferta de aconselhamento para atividade física e os fatores associados utilizando abordagens observacionais. Por outro lado, estudos de intervenção procuraram avaliar o efeito destas nos níveis de atividade física dos usuários e nas práticas de aconselhamento dos profissionais de saúde.

A prevalência de aconselhamento entre usuários de saúde variou entre 20,0\% (DURO et al., 2015) e 59,4\% (BARBOSA et al., 2017). Essa grande discrepância se deve aos diferentes objetivos, públicos-alvo, bem como à heterogeneidade na operacionalização davariável aconselhamento.Aparentemente, estudos comamostras recrutadas dentro das UBS (BARBOSA et al., 2017; LOPES et al., 2014; TOLEDO; ABREU; LOPES, 2013) apresentam maiores prevalências de aconselhamento comparadas àquelas de base domiciliar (DURO et al., 2015; SIQUEIRA et al., 2009), indicando, supostamente, um efeito positivo do trabalho das equipes de saúde da família com ações de educação em saúde. Subsidiando parcialmente esta hipótese, o estudo de Siqueira et al. (2009), comparando o recebimento de aconselhamento entre usuários atendidos em unidades de saúde com formato tradicional e aqueles atendidos no modelo ESF encontrou que entre estes o nível de aconselhamento foi maior.

Quando considerada a informação sob a perspectiva do aconselhador, a prevalência de aconselhamento para atividade física variou de 59,7\%, entre agentes comunitários de saúde (FLORINDO et al., 2015), a 68,9\% entre profissionais de enfermagem e medicina (FLORINDO et al., 2013). Os médicos relataram em maior proporção a realização do aconselhamento (81,2\%) (FLORINDO et al., 2013), bem como foram citados pelos usuários como os que mais aconselham (DURO et al., 2015; HALLAL et al., 2010; LOPES et al., 2014; SANTOS et al., 2012). Estes resultados sugerem que profissionais médicos incorporaram em suas práticas o aconselhamento para atividade física com maior frequência, sinalizando uma ampliação das práticas destes profissionais e um abandono de abordagens baseadas exclusivamente na medicalização. Por outro lado, indicam a necessidade de investimento em educação permanente dos demais trabalhadores, uma vez que se espera que qualquer profissional da equipe realize ações de educação em saúde, particularmente os agentes comunitários de saúde, devido ao fato de serem normalmente moradores das áreas sob sua responsabilidade, são talvez os mais indicados para identificar as reais necessidades de saúde da população (MOURA; SILVA, 2015).

Observou-se que o relato de aconselhamento foi mais comum em subgrupos populacionais, notadamente as pessoas que referiram DCNTs, a exemplo de obesos (DURO et al., 2015; HALLAL et al., 2010; LOPES et al., 2014), diabéticos e hipertensos (DURO et al., 2015; LOPES et al., 2014; SIQUEIRA et al., 2009). Considerando que a prática de atividade física pode ser um fator importante no tratamento destas condições, estes resultados são esperados. Destacam-se ainda as fortes evidências 
dos benefícios do aconselhamento para atividade física na prevenção primária de doenças crônicas não transmissíveis, particularmente as de origem cardiovascular (CURRY et al., 2014).

Outros subgrupos, como mulheres (DURO et al., 2015; HALLAL et al., 2010; SIQUEIRAetal., 2009) e pessoas mais velhas (DURO etal., 2015; HÄFELE; SIQUEIRA, 2016; HALLAL et al., 2010; LOPES et al., 2014), relataram mais frequentemente o recebimento de aconselhamento. Estes resultados, em certo grau, refletem o público de usuários da atenção básica no Brasil (BRASIL, 2015). Contudo, é importante ressaltar que o aconselhamento pode ser ofertado aos usuários independentemente da presença ou não de doenças, bem como do sexo ou idade, visto que a prática de atividade física pode ser benéfica para a maioria das pessoas (WHO, 2010).

Com relação ao conteúdo e forma do aconselhamento realizado, as investigações têm avaliado se os parâmetros gerais das recomendações de atividade física para a saúde (frequência, intensidade e duração) têm sido contemplados (FLORINDO et al., 2013; HÄFELE; SIQUEIRA, 2016). Os resultados sugerem que o aconselhamento para atividade física é pouco estruturado e não leva em consideração as recomendações de atividade física para a saúde (WHO, 2010). Apesar de parecer contraditório, acreditamos que isto não representa uma fragilidade per se, uma vez que as recomendações podem constituir um pano de fundo para a interlocução entre o profissional e o usuário, mas não precisa ser nem a única, nem a principal mensagem para eles.

Além da percepção de falta de conhecimento entre os profissionais de saúde (FLORINDO et al., 2013), outros fatores parecem interferir no processo de aconselhamento para os usuários. Florindo et al. (2013) encontraram que médicos e enfermeiros que realizam medidas da atividade física, que não relataram a falta de tempo como barreira e que referiram existir programas de promoção de atividade física na unidade de saúde foram mais prováveis de aconselhar atividade física. Em um modelo de aconselhamento amplamente utilizado (Modelo dos 5A`s: Assess, Advise, Agree, Assist, Arrange; ESTABOOKS; GLASGOW; DZEWALTOWSKI, 2003), não só a medida da atividade física, mas também das crenças, conhecimentos e habilidades motoras, constituem o primeiro componente de um aconselhamento estruturado. Conhecer o ponto de partida de uma intervenção educativa pode, portanto, não só auxiliar no estabelecimento de metas com o usuário, mas também contribuir para a longitudinalidade do cuidado deste.

Com relação à percepção de falta de tempo, este parece ser um fator limitante importante entre os profissionais de saúde para a realização do aconselhamento. Apesar do aconselhamento para atividade física poder ser realizado em um curto espaço de tempo em uma consulta rotineira, ao considerar as diferentes demandas do serviço de saúde (ex.: ações programáticas) e do usuário (ex.: demanda espontânea com queixas diversas), bem como a quantidade total de usuários a serem atendidos, atividades preventivas e educativas podem acabar sendo preteridas pelos profissionais. De fato, a quantidade de pacientes atendidos parece influenciar na capacidade de trabalho dos profissionais no que tange ao aconselhamento para atividade física (DIEHL et al., 2015). Estes achados apontam a necessidade de se 
discutir e pactuar o processo de trabalho, de maneira que ações de educação em saúde ocupem um espaço de destaque na rotina de trabalho de todos os profissionais, garantindo, portanto, um maior equilíbrio entre ações de promoção, prevenção, tratamento e reabilitação.

A existência de programas de atividade física na UBS foi outro fator associado ao aconselhamento entre médicos/enfermeiros (FLORINDO et al., 2013). Este dado é corroborado por Lopes et al., (2014), que verificaram que aqueles usuários que participavam do Programa Academia da Saúde (PAS) relataram receber mais aconselhamento. Municípios assistidos pelo PAS, criado com o objetivo de ampliar as ações de promoção da saúde e a integralidade do SUS, possuem mais uma oportunidade de referenciamento dos usuários, contudo, o número reduzido de polos resulta em baixa cobertura da população. Levantamento realizado em 2016 verificou que dos 2.849 municípios habilitados para o programa, apenas $27 \%$ possuíam polos em funcionamento (SÁ et al., 2016). Outras possibilidades podem e devem ser exploradas pelas equipes de saúde juntamente com os usuários, considerando as potencialidades e o contexto de cada território, bem como o enfrentamento das principais barreiras que se apresentem.

Dos estudos de intervenção incluídos, três avaliaram o impacto do aconselhamento nos níveis de atividade física dos usuários, e encontraram, de maneira geral, efeitos modestos (COSTA et al., 2015; GOMES; DUARTE, 2008; RIBEIRO et al., 2017). As estratégias variaram quanto a duração, intensidade, estratégia, pessoal envolvido e desfechos avaliados, o que limita comparações diretas entre elas. Ao analisar estas intervenções sob a perspectiva de aplicabilidade nos serviços de saúde, verificou-se que elas possuem diferentes níveis de validade externa. Ao tomar como referência as diretrizes do trabalho dos Núcleo de Apoio à Saúde da Família (NASF), atualmente denominado Núcleo Ampliado de Saúde da Família e Atenção Básica (NASF-AB) (BRASIL, 2017), é plausível prever que os papéis assumidos pelos pesquisadores nestes estudos sejam realizados pelos profissionais das equipes do NASF-AB. Contudo, ao considerar a intensidade e complexidade destas intervenções, devemos refletir sobre a capacidade de trabalho destes profissionais, que, conforme as diretrizes, devem apoiar de oito a 15 ESF (BRASIL, 2017). Diante disso, as atividades de apoio matricial do NASF-AB são fundamentais para viabilizar, por meio de suporte técnico-pedagógico para as equipes, o desenvolvimento dos processos de trabalho que tematizem a atividade física. Por outro lado, este movimento representaria uma aproximação à lógica do apoio matricial, e, ao mesmo tempo, uma abertura dos profissionais de Educação Física para a compreensão da atividade física/práticas corporais como objeto não exclusivo da área (LOCH; DIAS; RECH, 2019).

Um pouco mais alinhados com esta perspectiva, os demais estudos de intervenção tiveram como foco principal a formação dos profissionais da ESF para realizar aconselhamento entre os usuários (FLORINDO et al., 2015; SÁ; FLORINDO, 2012). De maneira geral, os resultados destas intervenções foram modestos em termos de mudança de práticas e percepções sobre adoção e aconselhamento de modos de vida saudáveis entre os profissionais (FIGUEIRA et al., 2015; MENDONÇA; 
TOLEDO; LOPES, 2015; SÁ; FLORINDO, 2012). Algumas intervenções adotaram o arcabouço teórico da pedagogia freireana, com estratégias que valorizaram a problematização e a construção contextualizada e coletiva de soluções, portanto, alinhadas com as diretrizes da Política Nacional de Educação Permanente (BRASIL, 2004). Contudo, ainda parece ser forte entre os profissionais de saúde a concepção biomédica, preventivista e de responsabilização individual dos usuários e dos próprios profissionais de saúde sobre a adoção de modos de vida saudáveis (FIGUEIRA et al., 2015; MENDONÇA; TOLEDO; LOPES, 2015; SÁ; FLORINDO, 2012).

O tempo e a intensidade limitada das intervenções podem parcialmente explicar os resultados. Uma mudança de perspectiva e de modos de fazer no cuidado à saúde exige o estabelecimento de aprendizagem significativa, produção de sentidos, autoanálise e autogestão (CECCIM, 2005), o que dificilmente foi alcançado em tão pouco tempo. Um possível caminho para a produção de sentidos e significados positivos em relação à atividade física entre os profissionais de saúde seria, por exemplo, oportunizar experiências de práticas corporais para este público. Esta se apresentou como demanda dos profissionais do SUS (FLORINDO et al., 2018), e certamente pode repercutir nas práticas de aconselhamento para os usuários.

A promoção de modos de vida saudáveis por meio do aconselhamento para atividade física na atenção primária à saúde permanece, portanto, como um desafio a ser empreendido por cada equipe de saúde e gestão local dos serviços. Contudo, é importante destacar que abordagens comportamentais têm potencial limitado na mudança de comportamentos de saúde quando descoladas da realidade do cotidiano das pessoas. $\mathrm{O}$ aconselhamento deve ser entendido como mais um modo de produção de cuidado na atenção primária, devendo estar articulado a ações de natureza intra e intersetoriais. Além disso, para que atividade física/práticas corporais componham os fazeres da produção de cuidado na atenção primária à saúde de acordo com os princípios do SUS, se faz necessário que a Educação Física, enquanto área de conhecimento e intervenção profissional, estabeleça uma aproximação mais qualificada e integrada com o campo de conhecimento da Saúde Coletiva ( $\mathrm{LOCH}$; RECH; COSTA, 2020).

\section{CONSIDERAÇÕES FINAIS}

Com base no exposto, elencamos como potencialidades para a realização do aconselhamento para atividade física na atenção primária em saúde: a compreensão da ESF como contexto privilegiado para ações de natureza educativa; os profissionais do NASF-AB como potencializadores das práticas de aconselhamento das equipes de referência; a existência de experiências prévias que podem ser ajustadas e experimentadas em diferentes realidades. Poroutrolado, elencamos como fragilidades: o predomínio da perspectiva biomédica e de responsabilização individual por parte dos profissionais de saúde; demandas do serviço e dificuldades de gerenciamento organizacional que dificultam a adoção de práticas de aconselhamento; deficiências na formação inicial e permanente que dificultam a realização qualificada e rotineira do aconselhamento para a prática de atividade física. 


\section{REFERENNCIAS}

ALY, Célia Maria C. et al. O Sistema Único de Saúde em série histórica de indicadores: uma perspectiva nacional para ação. Saúde Debate, v. 41, n.113, p. 500-512, 2017.

BARBOSA, Jessyka M. V. et al. Correlates of physical activity counseling by health providers to patients with diabetes and hypertension attended by the Family Health Strategy in the state of Pernambuco, Brazil. Primary Care Diabetes, v. 11, n. 4, p. 327-336, May 2017.

BRASIL. Ministério da Saúde. Avaliação das ações de aconselhamento em DST/Aids. Coordenação Nacional de DST e Aids. Brasília, DF: Ministério da Saúde, 1999.

BRASIL, Ministério da Saúde. Portaria n. 198/ GM/MS, de 13 de fevereiro de 2004. Institui a Política Nacional de Educação Permanente em Saúde como estratégia do SUS para a formação e o desenvolvimento de trabalhadores para o setor e dá outras providências. Brasília, DF: Ministério da Saúde, 2004.

BRASIL. Ministério da Saúde. Secretaria de Atenção à Saúde. Departamento de Atenção Básica. Portaria $\mathbf{n}^{\circ} \mathbf{2 . 4 3 6}$, de 21 de setembro de 2017. Aprova a política nacional de atenção básica, estabelecendo a revisão de diretrizes para a organização da atenção básica, no âmbito do sistema único de saúde (SUS). Brasília, DF: Ministério da Saúde, 2017.

BRASIL. Ministério da Saúde. Retratos da atenção básica no Brasil 2012. Satisfação dos usuários da atenção básica. Brasília: Ministério da Saúde, 2015.

BURDICK, Laura et al. Physicians', nurses' and community health workers' knowledge about physical activity in Brazil: A cross-sectional study. Preventive Medicine Reports, v. 2, p. 467-472, Jun. 2015.

CECCIM, Ricardo Burg. Educação Permanente em Saúde: desafio ambicioso e necessário. Interface - Comunicação, Saúde, Educação, v. 9, n. 16, p. 161-177, fev. 2005.

COSTA, Evelyn Fabiana et al. Avaliação da efetividade da promoção da atividade física por agentes comunitários de saúde em visitas domiciliares. Cadernos de Saúde Pública, v. 31, n. 10, p. 2185-2198, out. 2015.

CURRY, Susan J. et al. Behavioral Counseling Research and Evidence-Based Practice Recommendations: U.S. Preventive Services Task Force Perspectives. Annals of Internal Medicine, v. 18, n. 160, p. 407-414, Mar. 2014.

DIEHL, Katharina et al. Physical activity counseling by primary care physicians: attitudes, knowledge, implementation, and perceived success. Journal of Physical Activity and Health, v. 12, n. 10, p. $216-223$, Feb.2015.

DURO, Suele M. S. et al. Adult physical activity counseling by health professionals in Brazil: a national urban population survey. Journal of Physical Activity \& Health, v. 12, n. 8, p.1177 - 1183, Aug. 2015.

ESTABROOKS, Paul A.; GLASGOW, Russel E.; DZEWALTOWSKI, David A. Physical activity promotion through primary care. JAMA, v. 28, n. 22, p. 2913 - 2916, Jun. 2003.

FIGUEIRA, Taís. R. et al. Percepções sobre adoção e aconselhamento de modos de vida saudáveis por profissionais de saúde. Trabalho, Educação e Saúde, v. 13, n. 1, p. 181 200, jun. 2015.

FLORES, Thaynã R. et al. Aconselhamento por profissionais de saúde e comportamentos saudáveis entre idosos: estudo de base populacional em Pelotas, sul do Brasil, 2014.

Epidemiologia e Serviços de Saúde. v. 27, n. 1, p. e201720112, jun. 2018. 
FLORINDO, Alex A. et al. Association of knowledge, preventive counseling and personal health behaviors on physical activity and consumption of fruits or vegetables in community health workers Health behavior, health promotion and society. BMC Public Health, v. 15, n. 1, p. 1-8, Jan. 2015.

FLORINDO, Alex A. et al. Physical activity counseling in primary health care in Brazil: a national study on prevalence and associated factors. BMC Public Health, v. 13, n. 1, p. 1-10, Aug. 2013.

FLORINDO, Alex A. et al. Physical activity promotion by health practitioners: a distancelearning training component to improve knowledge and counseling. Primary health care research \& development, v. 19, n. 2, p.140 -150, Nov. 2018.

FLORINDO, Alex A. et al. Physical activity promotion in primary health care in Brazil: a counseling model applied to community health workers. Journal of Physical Activity and Health, v. 11, n. 8, p. 153-19, Nov. 2014.

FLORINDO, Alex A.; ANDRADE, Douglas R . Experiências de promoção da atividade física na estratégia de saúde da família. Florianópolis: Sociedade Brasileira de Atividade Física e Saúde, 2015.

GOMES, Marcius de Almeida; DUARTE, Maria F. S. Efetividade de uma intervenção de atividade física em adultos atendidos pela estratégia saúde da família: Programa Ação e Saúde Floripa. Revista Brasileira de Atividade Física \& Saúde, v.13, n. 1, p. 44-56, nov. 2008.

HÄFELE, Vítor; SIQUEIRA, Fernando V. Aconselhamento para atividade física e mudança de comportamento em Unidades Básicas de Saúde. Revista Brasileira de Atividade Física \& Saúde, v. 21, n. 6, p. 581-592, jan. 2016.

HALLAL, Pedro C. et al. Physical activity advice: short report from a population-based study in Brazil. Journal of Physical Activity \& Health, v. 7, n. 3, p. 352-354, Jan. 2010.

LOCH, Mathias R.; DIAS, Douglas F.; RECH, Cassiano R. Apontamentos para a atuação do Profissional de Educação Física na Atenção Básica à Saúde: um ensaio. Revista Brasileira de Atividade Saúde. v. 24, n. 1 p. 69 -73, ago. 2019.

LOCH, Mathias R.; RECH, Cassiano R.; COSTA, Filipe F. A urgência da Saúde Coletiva na formação em Educação Física: Lições com o COVID-19. Ciência e Saúde Coletiva, v. 25, n. 9, p. 3511-3516, set. 2020.

LOPES, Aline Cristina S. et al.Condições de saúde e aconselhamento sobre alimentação e atividade física na Atenção Primária à Saúde de Belo Horizonte-MG. Epidemiologia e Serviços de Saúde, v. 23, n. 3, p. 305-316, fev. 2014.

MENDONÇA, Raquel D.; TOLEDO, Mariana T. T.; LOPES, Aline C.S.; Incentivo à prática de aconselhamento sobre modos saudáveis de vida na Atenção Primária à Saúde. Escola Anna Nery - Revista de Enfermagem, v.19, n. 1 p. 140 - 146, mar. 2015.

MOURA, Raul F. S.; SILVA, Carlos. R.C. Afetividade e seus sentidos no trabalho do agente comunitário de saúde. Physis Revista de Saúde Coletiva. v. 25, n. 3, p. 993 - 1010, abr. 2015.

NICE - NATIONAL INSTITUTE FOR HEALTH AND CARE EXCELLENCE. Physical activity: brief advice for adults in primary care. London: NICE, May 2013.

RIBEIRO, Evelyn H. C. et al. Avaliação da efetividade de intervenções de promoção da atividade física no Sistema Único de Saúde. Revista de Saúde Pública. v. 51, n. 56, p. 1 12, 2017. 
SÁ, Gisele B. A. R. et al.O Programa Academia da Saúde como estratégia de promoção da saúde e modos de vida saudáveis: cenário nacional de implementação. Ciência \& Saúde Coletiva. v. 21, n. 6, p. 1849 - 1860, nov. 2016.

SÁ, Thiago H.; FLORINDO, Alex A. Efeitos de um programa educativo sobre práticas e saberes de trabalhadores da Estratégia de Saúde da Família para a promoção de atividade física. Revista Brasileira de Atividade Física \& Saúde, v. 17, n. 4, p. 293 - 299, jan 2012.

SÁ, Thiago H.; VELARDI, Marília.; FLORINDO, Alex A. Limites e potencialidades da educação dos trabalhadores de saúde da família para promoção da atividade física: uma pesquisa participativa. Revista Brasileira de Educação Física e Esporte, v. 30, n. 2, p. 417 - 426, fev. 2016.

SANTOS, Regiane P. et al. Aconselhamento sobre alimentação e atividade física: prática e adesão de usuários da atenção primária. Revista Gaúcha de Enfermagem, v. 33, n. 4, p. 14- 21, jun. 2012.

SANTOS, Taynã l. et al.Práticas pessoais e profissionais de promoção da atividade física em agentes comunitários de saúde. Revista Brasileira de Atividade Física \& Saúde. v. 20 , n. 2, p. 165- 73, jun. 2015.

SCHMIDT, Maria L. et al. Chronic non-communicable diseases in Brazil: burden and current challenges. The Lancet, v. 377, n. 97 p. 1949-1961, Aug. 2011.

SIQUEIRA, Fernando Vinholes et al. Aconselhamento para a prática de atividade física como estratégia de educação à saúde. Cadernos de Saúde Pública. v. 25, n. 1, p. 203 213, nov. 2009.

SOUZA, Marcela T.; SILVA, Michelly D.; CARVALHO, Rachel. Revisão integrativa: o que é e como fazer. Einstein (São Paulo), v. 8, n. 1, p. 102 - 106, fev. 2010.

TOLEDO, Mariana T. T.; ABREU, Mery N.; LOPES, Aline C. S.; Adesão a modos saudáveis de vida mediante aconselhamento por profissionais de saúde. Revista de Saúde Pública, v. 47 , n. 3 , p. $540-548$, fev. 2013.

TOLEDO, Mariana T. T. et al. Aconselhamento sobre modos saudáveis de vida na atenção primária à saúde. O Mundo da Saúde, v. 41, n.1, p. 87 - 97, jan. 2017.

WHO - WORLD HEALTH ORGANIZATION. Global recommendations on physical activity for Health. Geneva: WHO, 2010. 
Abstract: This study analyzes academic literature on counseling for physical activity in primary health care under Brazil's Unified Health System (SUS). This is an integrative literature review conducted on content from the LILACS, MEDLINE, PubMed and SciELO databases published until December 2018. Twenty-two studies were analyzed, of which 13 were observational, seven were experimental and two were qualitative. The prevalence of counseling varied from $20 \%$ to $59,4 \%$ between studies and the profiles of counseled patients were observed (women, elderly people, patients diagnosed with chronic diseases). Counseling is most often provided by doctors, physically active people, those who feel able to counsel, and people who do not see lack of time as a barrier. Interventions had modest effects on patients' physical activity levels and professionals' counseling practices.

Keywords: Counseling, Health education, Primary Health Care. Motor activity.

Resumen: El objetivo fue sintetizar la producción académica sobre el tema de la asesoría para la actividad física en la atención primaria de salud del Sistema Único de Salud (SUS). Se trata de una revisión integrativa de la literatura, realizada en las bases de datos LILACS, MEDLINE, PubMed y SciELO, publicada hasta diciembre de 2018. Se analizaron 22 estudios, de los cuales 13 fueron observacionales, siete intervenciones y dos cualitativos. La prevalencia de asesoría a usuarios varió del $20 \%$ al $59,4 \%$ entre los estudios y se observó el perfil de usuarios que más recibieron asesoría (mujeres, personas mayores y personas diagnosticadas con enfermedades crónicas). Los profesionales que más asesoran son: médicos, aquellos que son físicamente activos, que se sienten capaces de aconsejar y que no identifican la falta de tiempo como una barrera. Las intervenciones tuvieron efectos modestos en los niveles de actividad física de los usuarios y en las prácticas de asesoría de los profesionales.

Palabras clave: Asesoría. Educación en salud. Atención primaria de salud. Actividad motora. 


\section{LICENÇA DE USO}

Este é um artigo publicado em acesso aberto (Open Access) sob a licença Creative Commons 4.0 (CC BY), que permite uso, distribuição e reprodução em qualquer meio, desde que o trabalho original seja corretamente citado, sem restrição inclusive o uso para fins comerciais. Mais informações em: http://creativecommons. org/licenses/by/4.0

\section{CONFLITO DE INTERESSES}

Os autores declararam que não há conflito de interesses neste trabalho.

\section{CONTRIBUIÇÕES AUTORAIS}

João Miguel de Souza Neto: concepção do estudo, o levantamento, a análise e a interpretação dos dados e redigiram o manuscrito.

Geraldo Eduardo Guedes de Brito: concepção do estudo, revisão crítica dos conteúdos e aprovação da versão final.

Mathias Roberto Loch: concepção do estudo, revisão crítica dos conteúdos e aprovação da versão final.

Sanderson Soares da Silva: concepção do estudo, o levantamento, a análise e a interpretação dos dados e redigiram o manuscrito.

Filipe Ferreira da Costa: concepção do estudo, o levantamento, a análise e a interpretação dos dados e redigiram o manuscrito.

\section{FINANCIAMENTO}

O presente trabalho foi realizado sem qualquer apoio financeiro.

\section{COMO REFERENCIAR}

SOUZA NETO, João Miguel de; BRITO, Geraldo Eduardo Guedes de; LOCH, Mathias SILVA, Roberto Sanderson Soares da; COSTA, Filipe Ferreira da. Aconselhamento para atividade física na atenção primária à saúde: uma revisão integrativa. Movimento, v. 26, p. e26075, jan./dez. 2020. Disponível em: https:// seer.ufrgs.br/Movimento/article/view/104360 Acesso em: 28 nov. 2020. DOI: https://doi.org/10.22456/1982-8918.104360

\section{RESPONSABILIBADE EDITORIAL}

Alex Branco Fraga*, Elisandro Schultz Wittizorecki, Ivone Job*, Mauro Myskiw*, Raquel da Silveira*

*Universidade Federal do Rio Grande do Sul, Escola de Educação Física, Fisioterapia e Dança, Porto Alegre, RS, Brasil 